\title{
ACCELERATION OF HIGH INTENSITY PROTON BEAMS
}

X. Altuna, G. Arduini, C. Arimatea, R. Bailey, R. Billen, T. Bohl, P. Collier, K, Cornelis, G. Crockford, B. Desforges, C. Despas, A. Faugier, A. Ferrari, R. Giachino, K. Hanke, M. Jonker, T. Linnecar, C. Niquille, L. Normann, G. Robin, G. Roy.

\section{CERN}

Geneva, Switzerland

\section{Abstract}

In 1998 the CERN SPS accelerator finished a five years long program providing $450 \mathrm{GeV}$ proton beams for neutrino physics. These experiments required the highest possible beam intensity the SPS can deliver. During the last five years the maximum proton intensity in the SPS has steadily been increased to a maximum of $4.810^{13}$ protons per cycle. In order to achieve these intensities a careful monitoring and improvement of the vertical aperture was necessary. Improved feedback systems on the different RF cavities were needed in order to avoid instabilities. Also the quality (emittance and extraction spill) of the injector, the CERN PS, had be optimised.

\section{GENERAL LAYOUT OF THE SPS CYCLE.}

In Fig 1 a typical SPS cycle is shown as it was used for the last five years during the high proton intensity running:

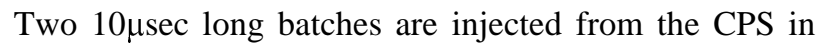
order to fill the whole ring. The injection energy is 14 $\mathrm{GeV}$. The two injections come in with a $1.2 \mathrm{sec}$ time interval.

The beam is then ramped up to $450 \mathrm{GeV}$, crossing transition at $23.6 \mathrm{GeV}$. The maximum intensity accelerated up to now is $4.8610^{13}$ protons/cycle.

At $445 \mathrm{GeV}$ a first fast resonant extraction (FRE1) takes place. An intensity, up to $1.810^{13}$ protons, is extracted on the half integer, in straight section 6 (fig 2). The spill (fig 3), $6 \mathrm{msec}$ long, is controlled by a quadrupole kicker. The pulse shape of this kicker is controlled by an IGBT (Insulated Gate Bipolar Transistor) in order to obtain an as flat as possible maximum [1]. This beam is sent to the neutrino experiment.

At $450 \mathrm{GeV}$, a shared slow resonant extraction (SSRE) on the third integer, takes part of the beam out in straight section 2 and 6 . The phase advance between 6 and 2 is put exactly set to 9 betatron oscillations in the horizontal plane. The spill length is $2.3 \mathrm{sec}$ and the extracted intensity can go up to $210^{13}$ (fig 4).

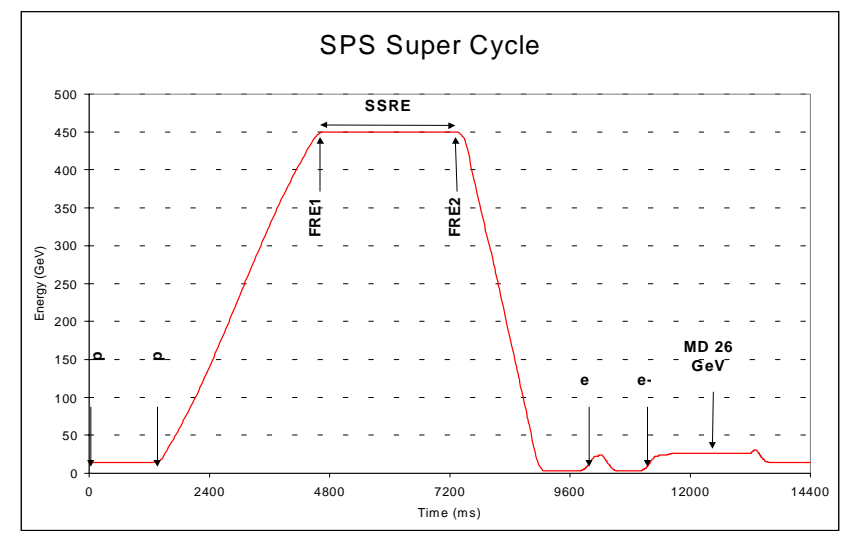

Fig 1 : The SPS supercycle

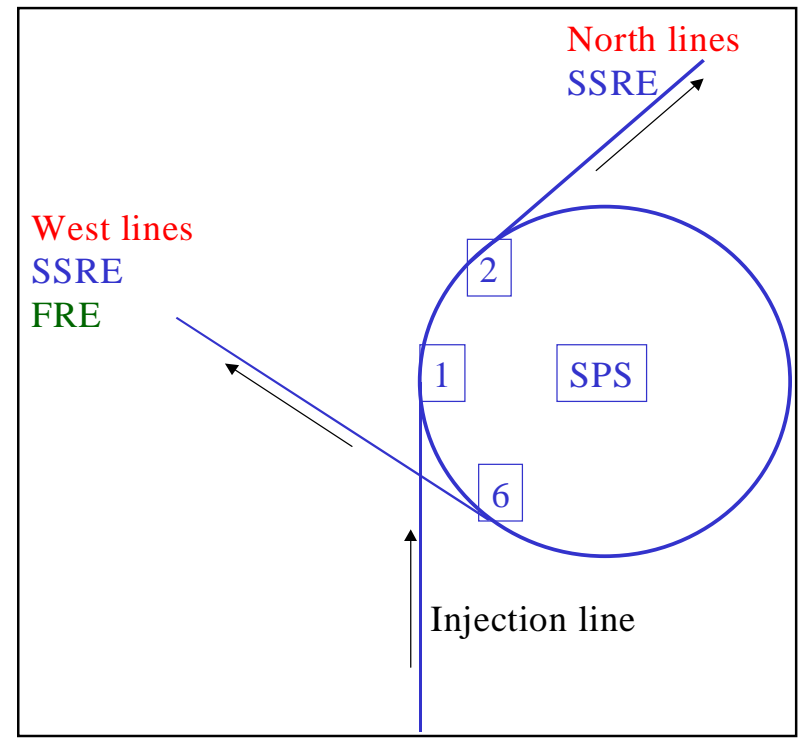

Fig 2 : Schematic layout of the SPS. 
The remaining beam is extracted for neutrino physics by a second fast resonant extraction (FRE2) on the half integer, in straight section 6 . The spill is $6 \mathrm{msec}$ long and the extracted intensity can go up to $1.810^{13}$ protons.

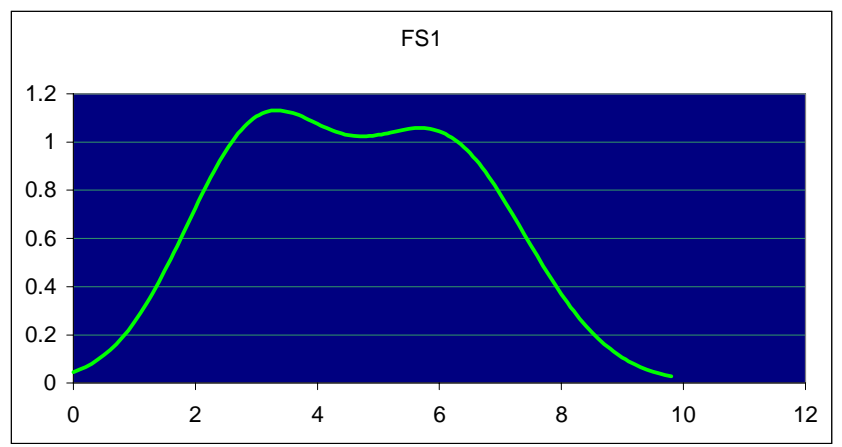

Fig 3 : Typical spill of the fast resonant extraction.

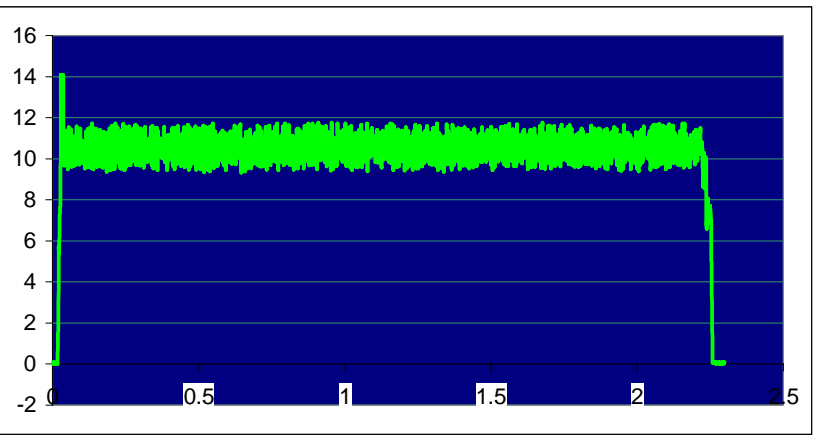

Fig $4:$ spill off the shared slow extraction $(2.3 \mathrm{sec}$ long).

After the proton physics cycle there are two LEP injection cycles $(3.5 \mathrm{GeV}-22 \mathrm{GeV}$ ) for positrons and electrons.

At the end there is a $26 \mathrm{GeV}$ injection plateau which is used for machine studies on the future LHC beam.

\section{MAJOR STEPS TO INCREASE THE PROTON INTENSITY.}

Since 1993 the intensity of the SPS proton beam has increased from $3.510^{13}$ protons /cycle to $4.8610^{13}$ protons /cycle. This progress was achieved by the following measures:

- $\quad$ The proton intensity per CPS pulse was raised to 3 $10^{13}$ protons per cycle

- The CPS improved the control of the extracted beam (5 turn extraction) in order to avoid peaks in the spill which give rise to very harmful peak power in the feedback for the superconducting cavities. These cavities are used for lepton acceleration and are actively damped during the proton cycle.

- A better "alignment" of the five extracted turns in the CPS could be obtained, so that they arrive with the same angle and position in the SPS, improving the overall emittance of the injected beam.

- The vertical aperture of the SPS was continuously monitored with bump scans and beam loss analysis. The bottle necks could be removed by re-aligning certain elements and exchanging magnets with distorted vacuum chambers inside. The aperture increased form $21 \mathrm{~mm}$ to $37 \mathrm{~mm}$ (at $\beta=100 \mathrm{~m}$ ) over the last five years.

- Thanks to a yearly realignment program of the quadrupoles, the natural closed orbit distortion (without corrections) could be reduced to $2.3 \mathrm{~mm}$ rms in the horizontal plane and $1.2 \mathrm{~mm} \mathrm{rms}$ in the vertical plane. Hence less corrector strength is needed and the closed orbit correction could be extended form $14 \mathrm{GeV}$ to $50 \mathrm{GeV}$ resulting in reduced losses at transition.

- The super conducting cavities that are used for lepton acceleration were more efficiently damped, using a feed forward loop and a one-turn feedback [2].

- The betatron- and dispersion mismatch at injection were carefully measured and could be reduced to less than $10 \%$ [3].

- Another problem with high intensities is the frequent sparking of the electrostatic septa. The ion traps inside the septa had to be trimmed carefully to empirical values in order to reduce the spark rate.

All these measures improved the overall transmission in the machine and a record intensity of $4.8610^{13}$ could be reached. The loss pattern in the optimised machine is the following :

- $8 \%$ to $12 \%$ losses at CPS extraction.

- $\quad 0 \%$ to $2 \%$ losses at injection in SPS.

- $2 \%$ losses at start of acceleration (capture losses).

- $2 \%$ to $3 \%$ losses at transition.

- $1 \%$ to $2 \%$ losses at extraction

- $5 \%$ to $10 \%$ losses splitting and transfer to targets for the neutrino beam there is no splitting, and the transfer losses are negligible.

The gradual improvement over the last years can be seen form fig 5 where the total number of protons delivered to all targets is plotted. The total number of protons on the neutrino target is shown in fig 6 . 


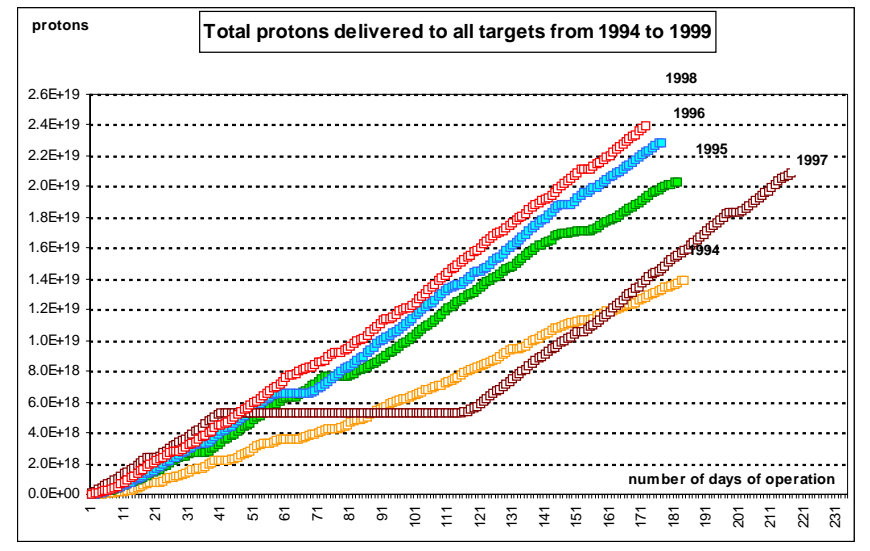

Fig 5 : Total proton intensity on targets for the last five years. The long period without production in 1997 was due to a fire in BA3.

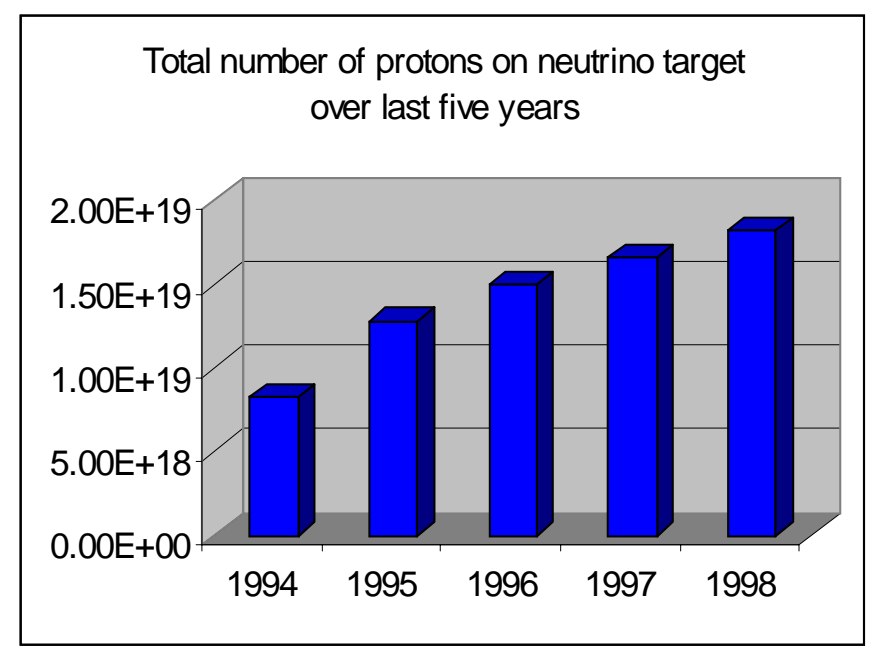

Fig 6 : total intensity on the neutrino target over the last years.

\section{REFERENCES}

[1] E. Carlier, A. Heinemann, E. Vossenberg, Electronic regulation of the SPS extraction quadrupole current pulse shape for improved stability of the extracted beam, CERN-SL /note 96-78 (1996)

[2] D. Boussard, G. Lambert, T. Linnecar, PAC -1993.

[3] G. Arduini, M. Giovannozzi, K. Hanke, D. Manglunki, M. Martini, G. Metral, Measurement and Optimization of the PS-SPS Transfer Line Optics, these proceedings. 